\title{
The Key Frame Extraction Algorithm Based on the Indigenous Disturbance Variation Difference Video
}

\author{
Zhang Ronghua and Liu Changzheng*
}

College of Information Science and Technology, Shihezi University, Shihezi, 832003, China

\begin{abstract}
In view of the traditional support vector machine (SVM) learning algorithm's learning parameters are not easily determined in the process of video key frame extraction and the accuracy is low. An independent perturbation variable difference SVM algorithm is used for video key frame extraction. First of all, the biological mechanism of differential evolution algorithm was analyzed, and then an improved way of autonomic disturbance variation was put forward. Secondly, combined with improved forms of independent disturbance differential evolution algorithm for SVM parameters optimization, the key frame extraction algorithm of the video based on the improved differential of SVM algorithm was designed. The standard test functions and video test database experiment show that the improvement of autonomic disturbance variation difference video key frame extraction algorithm can optimize parameters of support vector machine more effectively and contribute to the improvement of video retrieval.
\end{abstract}

Keywords: Autonomic disturbance variation, Differential evolution, Video, Key frames, Extract.

\section{INTRODUCTION}

Video information extraction algorithms can be grouped into two types. One is based on the extraction of shot contents, and the other is based on the extraction of text description. The current commercial video key frame extraction method is based on text description. Text description extraction algorithm is relatively simple, which uses text retrieval to replace the video retrieval, and simplifies the calculation and complexity. The drawback of the method is: simplified text description often cannot accurately reflect the content of the video. The understanding of text meaning is different from person to person. It cannot use the existing image template or key frames characteristics, such as advanced retrieval methods. Under this background, the relevant researchers studied the shot content based on the video key frame extraction method (CBVR) [1].

To simplify the data processing, data frame processing mode of the video is converted to video retrieval in the video key frame extraction. The content-based video key frame extraction research has three mainstream research directions: One is the research of video feature extraction based on bottom, such as the literature [2] proposed a kind of color histogram key frame feature processing method, which reduce interference factors by removing the color. The second is the matching research based on video sample, such as the literature [3] based on Markov chain with dynamic characteristics, proposed a kind of graph template based on video key frame extraction system framework. These two

*Address correspondence to this author at the College of Information Science and Technology, Shihezi University, Shihezi, 832003, China; E-mail: liucz@sina.cn kinds of video key frame extraction methods have been very matured and used by commercial. The third direction is based on the semantic video key frame extraction, such as the literature [4] based on the movement of video semantic content modeling in this paper, a detection model of video semantic. Non-semantic of the bottom characteristics and the template matching method for high-level semantic concepts in human thinking are quite different. Bad understanding can affect the usability of video key frame extraction system. The method of key frame extraction based on semantic and non-semantic of the fundamental differences in the former uses the sample training of this machine learning method, and often uses main pattern recognition methods, learning algorithm such as MRF, SVM, etc.

Support Vector Machine (SVM) at the beginning of the design is mainly aimed at solving nonlinear problems such as small sample. The SVM theory gradually improves the learning method. Literature [5] designed gradient descent way for the SVM parameters selection. The gradient is a function value which increases or decreases the fastest direction, to improve the algorithm by using gradient approach has a lot of disadvantages. But the gradient being essentially a method of linear search is dependent on the initial value selection of SVM parameters, with the improvement of the performance of the algorithm of limitations. Literature [6] used the PSO of the nonlinear search features combined with $\mathrm{k}$-fold error algorithm for the SVM parameters selection, and the effect was very good.

Applying the intelligent optimization algorithm to the SVM parameters selection is a major innovation. The paper proposed an improved self perturbation mutation differential evolution based SVM algorithm (referred to as "ISPMDE- 
SVM"). We apply the algorithm to the video key frame extraction for laboratory studies.

\section{VIDEO FEATURE EXTRACTION AND PROCE- SSING}

\subsection{Motion Feature Extraction}

Literature [7] presented a motion feature extraction algorithm of video key frames (shown in Fig. (1)), $N_{1}, N_{2}, C_{1}, C_{2}$ as constant value factors. The main effect is the size of the calibration video key frames feature area. The left of the figure is a global motion feature extraction areas, the right of the figure is local motion feature extraction areas.
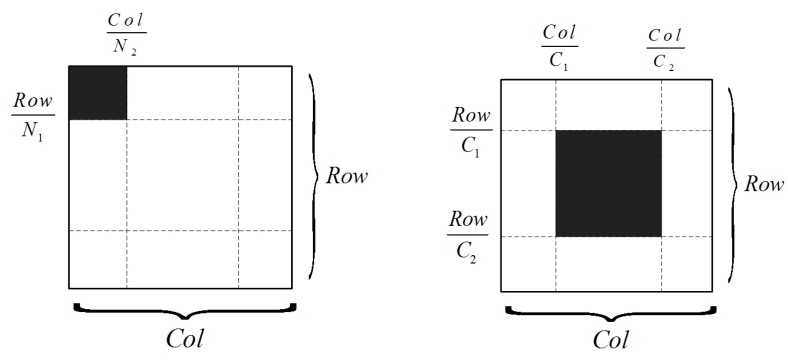

Fig. (1). Motion feature extraction area.

Global motion feature extraction: using LUCAS \& KANADE optical flow algorithm, the global motion characteristics of the frame of the video are as follows:

$G d(k)=\frac{\sum_{i=0}^{N_{1}} \sum_{j=0}^{N_{2}} g d(i, j)}{N_{1} \times N_{2}}$

Where $\operatorname{gd}(i, j)$ shows pixel $(i, j)$ of the optical flow size. The global motion characteristics of the distance between the $k, k+1$ frame are as follows:

$G D(k, k+1)=|G d(k)-G d(k+1)|$

Partial motion feature extraction: color histogram method is simple and it is similar to the human visual system. This article uses the space model calculating the local characteristics. Assume select $H 8, S 3, V 3$ divisions, the shot key frames can be converted into $72 \mathrm{~d}$ data array, and related algorithm used for the joining together of vector $[8 \sim$ 10]:

$l=9 H+3 S+V$

Vector $l[k, 0]: l[k, 71]$ is the HSV figure of images, that is, the frame of local motion characteristic [9]. The local movement distance between the $k$ frame and the $k+1$ frame can be defined as:

$$
P D(k, k+1)=\frac{\sum_{i=0}^{71}(l[k, i]-l[k+1, i])^{2}}{\left(\frac{\text { Col }}{C_{2}}-\frac{\text { Col }}{C_{1}}\right)+\left(\frac{\text { Row }}{C_{2}}-\frac{\text { Row }}{C_{1}}\right)}
$$

\subsection{Feature Vector Structure and Preprocessing}

After obtaining local and global motion characteristics, we can construct characteristic vector for:

$x[i]=\left(R_{1}, R_{2}, S_{1}, S_{2}, F\right)$

$F=n-m$ shows the shot interval number, $m, n$ shows the position between adjacent two frames. $R_{1}$ shows the two frames distance average value, $R_{2}$ shows the extent of change as a local motion between the two frames. $S_{1}$ shows the global movement feature in the distance average value between two key frames; $S_{2}$ shows two key frames global movement volatility situation. Define [6]:

$$
\left\{\begin{array}{l}
R_{1}=\frac{\sum_{i=0}^{n-m-1} P D(m+i, m+i+1)}{n-m} \\
R_{2}=\frac{\sum_{i=0}^{n-m-1}\left|R_{1}-P D(m+i, m+i+1)\right|}{n-m} \\
S_{1}=\frac{\sum_{i=0}^{n-m-1} G D(m+i, m+i+1)}{n-m} \\
S_{2}=\frac{\sum_{i=0}^{n-m-1}\left|S_{1}-G D(m+i, m+i+1)\right|}{n-m}
\end{array}\right.
$$

To obtain the video feature vector for normalization processing, construct the fitness function $f_{i}$ as follows:

$$
f_{i}=\beta_{1}\left(\alpha_{1} \frac{R_{1 x i}}{R_{1 A}}+\alpha_{2} \frac{R_{2 x i}}{R_{2 A}}\right)+\beta_{2}\left(\alpha_{1} \frac{S_{1 x i}}{S_{1 A}}+\alpha_{2} \frac{S_{2 x i}}{S_{2 A}}\right)
$$

where $R_{1 x i}$ is the vector $x[i]$ component values $R_{1}$, the rest is similar. $A, \beta$ are the weight values, $\alpha_{1}+\alpha_{2}=1, \beta_{1}+\beta_{2}=1$. If $R_{1 x i} / R_{1 A}$ 's four ratio value are greater than 1 , then takes its countdown.

\section{THE INDEPENDENT PERTURBATION MUTA- TION DIFFERENTIAL EVOLUTION ALGORITHM}

\subsection{Autonomic Disturbance Variation Way}

The variation algorithm of the original differential evolution algorithm is as follows:

$$
\begin{aligned}
& x_{m}=x_{s 3}^{s}+F *\left(x_{s 1}^{s}-x_{s 2}^{s}\right) \\
& x_{m}=x_{b}^{s}+F\left[\left(x_{s 1}^{s}-x_{s 2}^{s}\right)+\left(x_{s 3}^{s}-x_{s 4}^{s}\right)\right]
\end{aligned}
$$

The mutation method of the formula (8) is called $\mathrm{DE} / \mathrm{rand} / 1 / \mathrm{bin}$, and the mutation method of the formula (9) is called $\mathrm{DE} / \mathrm{best} / 2 / \mathrm{bin}$. In the two formulas, $x_{s 1}^{s}, x_{s 2}^{s}, x_{s 3}^{s}, x_{s 4}^{s}$ shows the different random particles. $x_{\mathrm{b}}^{s}$ shows the best position of this generation population individual. $F \in[0,2]$ shows the difference variable of scaling factor [4]. 
Relative to the formula (9), the formula (8) used three random variation ways of individual $x_{s 1}^{s}, x_{s 2}^{s}, x_{s 3}^{s}$. The selection method can maintain the diversity of population is suitable for global search, but the convergence speed is slow. While the formula (9) uses the current population of the best fitness individual $x_{\mathrm{b}}^{s}$ as basic variables, and uses four different random individuals $x_{s 1}^{s}, x_{s 2}^{s}, x_{s 3}^{s}, x_{s 4}^{s}$ as direction quantity to increase the variation individual of randomness, but the mutation is found in the simulation method, although the algorithm convergence speed is quick in the early stages, but falls into local extreme value points easily, which is relatively suitable for local search.

In conclusion, if a new mutation method is able to synthesize formula (8) and (9) mutation methods of the characteristics, the performance of the algorithm will be greatly improved. Literature [7] presented a double group pseudo parallel differential evolution algorithm. The algorithm adopts two populations parallel evolutionary. One population used the variation method of the formula (1), another population used the variation method of the formula (2); under certain conditions, two populations exchange information with each other. But it needs to be observed that he algorithm turns double populations algorithm complexity into two times, in exchange for the improvement of the performance. Formula (9) in the form of variation method, with four different random individual $x_{s 1}^{s}, x_{s 2}^{s}, x_{s 3}^{s}, x_{s 4}^{s}$ as the direction of quantity, can increase the mutated individual randomness, is helpful for maintaining the differences between individuals, which can maintain the diversity of population, so this article in the form of formula (9) is improved to:

$x_{i}^{s+1}=x_{i}^{s}+F\left(x_{r 1}^{s}-x_{i}^{s}+x_{r 2}^{s}-x_{r 3}^{s}\right)$

In formula (10), $x_{s 1}^{s}, x_{s 2}^{s}, x_{s 3}^{s}$ show random individual which are not same. Compared with mutation methods of formula (8) and formula (9), formula (10) takes $x_{i}^{s}$ as the base variable and takes it as a parameter of direction quantity. That is to say, individual variation is based on its own change, and maximum degree can keep the diversity of population.

In Fig. (2), “०"shows the $s$ generation of individuals, " "shows the $s+1$ generation of the individual, "ø" shows the local optimal value, “兯” shows the global optimal value. Fig. (2) shows that the independent disturbance (SPM) variation method has better ability of population diversity. Its offspring solution of the search area is the largest. $\mathrm{DE} / \mathrm{rand} / 1 / \mathrm{bin}$ is based on the expression of the random variation effect. The entire population near some individuals has more offspring individuals. Its ability to keep individual diversity is inferior to SPM. The DE/best/2/bin for each individual with $x_{\mathrm{b}}^{s}$ is based on individuals round $x_{\mathrm{b}}^{s}$. If $x_{\mathrm{b}}^{s}$ is a global optimal value, the algorithm can converge. If $x_{\mathrm{b}}^{s}$ is local minima value, the algorithm will be premature.

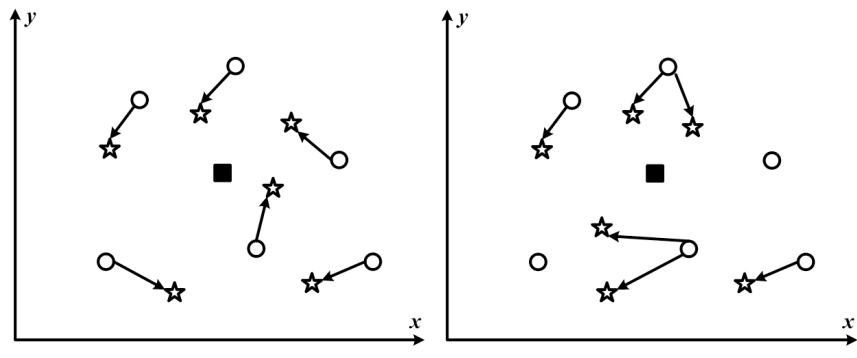

(a) ISPM mutation methods

(b) Formulas (8) variation methods

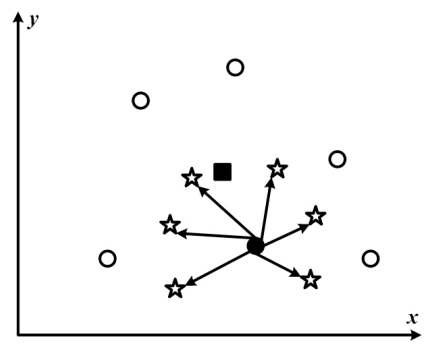

(c) Formulas (9) variation methods

Fig. (2). Three variation methods of individual distribution.

\subsection{Improvement of independent disturbance variation}

Although SPM mutation method has better ability to keep population diversity, but it emphasized on the population diversity excessively and ignored the population convergence speed. In order to balance this problem, for the selection of $x_{r 1}^{s}, x_{r 2}^{s}, x_{r 3}^{s}$, improved ways are put forward. From the perspective of vector operation, there is a problem of the vector direction. Generally, the fitness of the individual direction will be easier and faster to find the global optimal solution [7]. The SPM variation pattern will take sort ways as follows:

$$
\begin{aligned}
& x_{i}^{s+1}=x_{i}^{s}+F\left(x_{r b}^{s}-x_{i}^{s}+x_{r m}^{s}-x_{r w}^{s}\right) \\
& x_{r b}^{s}=\operatorname{best}\left(x_{r 1}^{s}, x_{r 2}^{s}, x_{r 3}^{s}\right) \\
& x_{r m}^{s}=\operatorname{mid}\left(x_{r 1}^{s}, x_{r 2}^{s}, x_{r 3}^{s}\right) \\
& x_{r w}^{s}=\operatorname{worst}\left(x_{r 1}^{s}, x_{r 2}^{s}, x_{r 3}^{s}\right)
\end{aligned}
$$

From vector angle consideration, $\left(x_{r b}^{s}-x_{i}^{s}+x_{r m}^{s}-x_{n v}^{s}\right)$ includes $x_{i}^{s}$ item, $x_{r b}^{s}$ as $x_{r 1}^{s}, x_{r 2}^{s}, x_{r 3}^{s}$ of the best individual, the adaptive value should be better than or equal to $x_{i}^{s}$, namely the selection $x_{r 1}^{s}, x_{r 2}^{s}, x_{r 3}^{s}$, first of all one of the fitness value is better than that of $x_{i}^{s}$. In order to improve the direction of the mutation operation, we balance the disadvantages factors. Due to the individual variation, the certainty is a random variable. It can guarantee the global search performance with efficient mutation. 


\subsection{ISPMDE Algorithm Steps}

Step 1: Set population number $N P$, scaling factor $F$, such as algorithm parameters, the initial search space $\left[l^{0}, u^{0}\right]$, $s=1$.

Step 2: Initial population and judge the individual adaptation degree.

Step 3: If meet the requirements of the terminating or precision grade, then terminate the algorithm.

Step 4: In the population randomly selected one by one individual $x_{r 1}^{s}$, satisfy the adaptive value $f\left(x_{r 1}^{s}\right) \leq f\left(x_{i}^{s}\right)$, again select $x_{r 2}^{s}, x_{r 3}^{s}$ randomly, meet $x_{r 1}^{s} \neq x_{i}^{s} \neq x_{r 2}^{s} \neq x_{r 3}^{s}$.

Step 5: Sort $x_{r 1}^{s}, x_{r 2}^{s}$ and $x_{r 3}^{s}$, determine the value of the $x_{r b}^{s}$, $x_{r m}^{s}$ and $x_{r w}^{s}$. Use formula (4) ISPMDE variation for population variation.

Step 6: Taking the crossover and selecting operation.

Step 7: Calculate the aim function value of the new species individual, make $s=s+1$, turn to Step3.

As you can see from the above steps, for the improvement of the standard differential evolution algorithm is just two steps in the Step4 and Step5. Since the standard differential evolution algorithm is mainly concentrated in the crossover operation, the effect of ISPMDE algorithm for time complexity is relatively smaller.

\section{ISPMDE-SVM KEY FRAME EXTRACTION PRO- CESS}

The extraction algorithm based on the ISPMDE-SVM video key frame process is shown in Fig. (3), assuming that the original sample video after the key frame extraction and the bottom feature extraction operation (see chapter 2), getting basic training data sets for:

$U=\left\{\left(x_{1}, y_{1}\right),\left(x_{2}, y_{2}\right), \cdots,\left(x_{n}, y_{n}\right)\right\}$

Using the formula (12) the video training data $U$ is combined with ISPMDE algorithm. We then use the SVM model to detect new video streaming. According to the output value of the SVM model (fitness value) we sort the video database for the user query. The process has three input parts: One is the video sample input, used as training data to ISPMDE-SVM parameter optimization. The second is video streaming input, used for SVM parameters detection and correction the SVM parameters optimization value. The third is the user query input, according to the user in the client input information for video database based on semantic query. In conclusion, the core part of the algorithm framework is the SVM parameters optimization. [11 12].

Literature [6] used k-fold as target value of parameter selection. The ISPMDE-SVM algorithm is shown:

Step 1: Reading the video sample data $S$, and random initialization $\{C, \phi\}$ as the initial values of differential evolution individuals.

Step 2: Taking the sample $S$ divided into $k$ individuals which do not include with each other of subset $S_{1}, \cdots, S_{k}$.

Step 3: Taking the above subset as training data set, using ISPMDE algorithm optimized the SVM algorithm parameter $\{C, \phi\}$, and calculating the k-fold cross-validation error as species adapt value $f$.

Step 4: Taking the operation steps related to difference, and using the ISPM mode for individual variation, which is given in this paper.

Step 5: Return to Step2, until it reaches the terminating number or meet the precision requirement.

\section{EXPERIMENTAL RESULTS AND ANALYSIS}

Experiment 1: (ISPMDE algorithm performance test) adopts the common practice to choose three commonly used basic test functions as the test object:

(1) $f 1=\sum_{i=1}^{30} x_{i}^{2}, \quad\left|x_{i}\right| \leq 100, \min (f 1)=0$

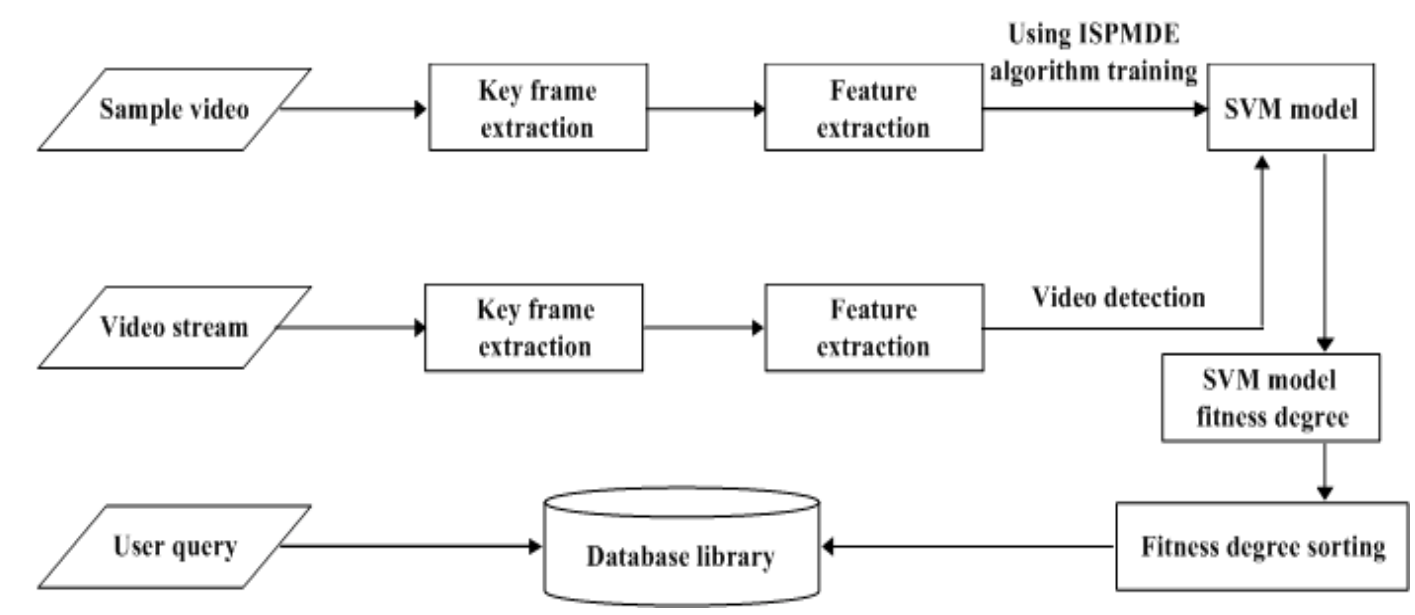

Fig. (3). ISPMDE-SVM key frame extraction. 
(2) $f 2=\frac{1}{4000} \sum_{i=1}^{30} x_{i}^{2}-\prod_{i=1}^{30} \cos \left(x_{i} / \sqrt{i}\right)+1$,

$\left|x_{i}\right| \leq 100, \min (f 2)=0$

(3) $f 3=\sum_{i=1}^{29}\left|100\left(x_{i+1}-x_{i}^{2}\right)^{2}+\left(x_{i}-1\right)^{2}\right|$,

$\left|x_{i}\right| \leq 5.12, \min (f 3)=0$

With reference to relevant literature and common standards, set up the simulation parameters as follows: the dimension $D=30$, population takes setting dimension of $5 \sim 10$ times ${ }^{[4]}$ in general, and the simulation of algorithm takes population $N P=200$. Parameters in ref. [4] $F \in[0.4,0.8], \quad C R \in[0.3,0.9]$. Set up the parameter [8] ASMDE and SACPMDE, DERL parameters [4], the standard DE algorithm $F=0.6, C R=0.8$, the simulation accuracy $V T R=10^{-6}$. Simulation results such as Table $\mathbf{1}$, as shown in Figs. (4-6). In order to compare the results, Figs. (4-6) show the convergence value of logarithmic mapping. Taking the function $f 4$ the convergence curve is opposite to logarithmic mapping.

Simulation experiment data in Table $\mathbf{1}$ and convergence curve in Figs. (4-6) shows:

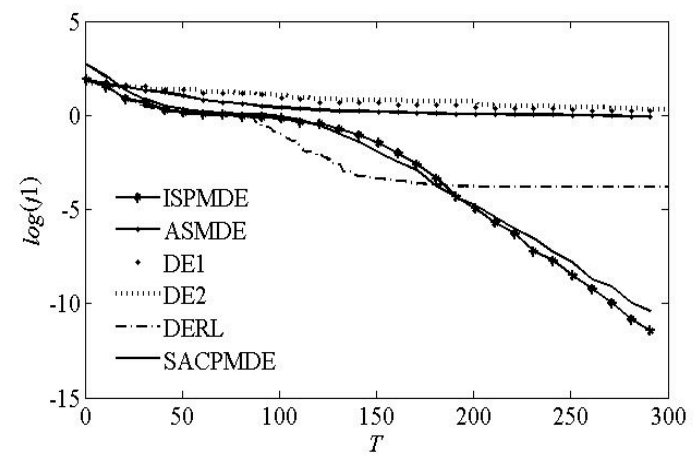

Fig. (4). Convergence curves of the objective function $f 1$.

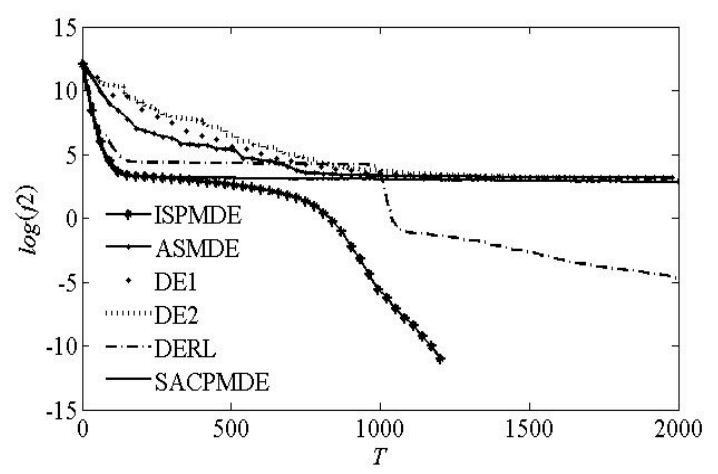

Fig. (5). Convergence curves of the objective function $f 2$.

Table 1. All algorithms' run averaging 20 times.

\begin{tabular}{|c|c|c|c|c|c|c|}
\hline & & $\begin{array}{c}\text { Optimal } \\
\text { Performance }\end{array}$ & $\begin{array}{l}\text { The Average } \\
\text { Performance }\end{array}$ & Iterations Number & Variance & Time/s \\
\hline \multirow{5}{*}{$f 1$} & ISPMDE & $5.24 \times 10^{-6}$ & $6.54 \times 10^{-6}$ & 331 & $2.69 \times 10^{-18}$ & 9.8 \\
\hline & SACPMDE & $5.37 \times 10^{-6}$ & $7.85 \times 10^{-6}$ & 297 & $8.77 \times 10^{-18}$ & 7.6 \\
\hline & ASMDE & $5.28 \times 10^{-6}$ & $7.76 \times 10^{-6}$ & 1087 & $8.21 \times 10^{-18}$ & 8.3 \\
\hline & $\mathrm{DE} / \mathrm{rand} / 1 / \mathrm{bin}$ & $6.67 \times 10^{-6}$ & $7.98 \times 10^{-6}$ & 2178 & $4.14 \times 10^{-13}$ & 21.0 \\
\hline & DE/best/2/bin & $6.42 \times 10^{-6}$ & $8.02 \times 10^{-6}$ & 2363 & $3.80 \times 10^{-13}$ & 23.5 \\
\hline \multirow{4}{*}{$f 2$} & ISPMDE & $4.57 \times 10^{-6}$ & $5.93 \times 10^{-6}$ & 306 & $3.52 \times 10^{-14}$ & 8.7 \\
\hline & SACPMDE & $5.98 \times 10^{-6}$ & $7.00 \times 10^{-6}$ & 301 & $5.16 \times 10^{-13}$ & 7.3 \\
\hline & $\mathrm{DE} / \mathrm{rand} / 1 / \mathrm{bin}$ & $6.64 \times 10^{-6}$ & $2.78 \times 10^{-4}$ & 2170 & $2.73 \times 10^{-6}$ & 25.1 \\
\hline & $\mathrm{DE} / \mathrm{best} / 2 / \mathrm{bin}$ & $6.86 \times 10^{-6}$ & $5.28 \times 10^{-3}$ & 5110 & $6.05 \times 10^{-5}$ & 67.3 \\
\hline \multirow{6}{*}{$f 3$} & ISPMDE & $5.78 \times 10^{-6}$ & $6.18 \times 10^{-6}$ & 1219 & $5.63 \times 10^{-13}$ & 35.3 \\
\hline & SACPMDE & $6.62 \times 10^{-6}$ & 1.35 & 4413 & $1.81 \times 10^{1}$ & 46.1 \\
\hline & ASMDE & $2.67 \times 10^{-3}$ & $6.31 \times 10^{-3}$ & 8000 & $8.51 \times 10^{-6}$ & 82.3 \\
\hline & DERL & $7.62 \times 10^{-6}$ & $1.41 \times 10^{2}$ & 6457 & $2.42 \times 10^{5}$ & 75.9 \\
\hline & $\mathrm{DE} / \mathrm{rand} / 1 /$ bin & $7.57 \times 10^{-6}$ & $7.92 \times 10^{-6}$ & 6170 & $1.19 \times 10^{-12}$ & 63.9 \\
\hline & DE/best/2/bin & $6.58 \times 10^{-6}$ & $1.99 \times 10^{-1}$ & 5970 & $6.95 \times 10^{-1}$ & 42.3 \\
\hline
\end{tabular}




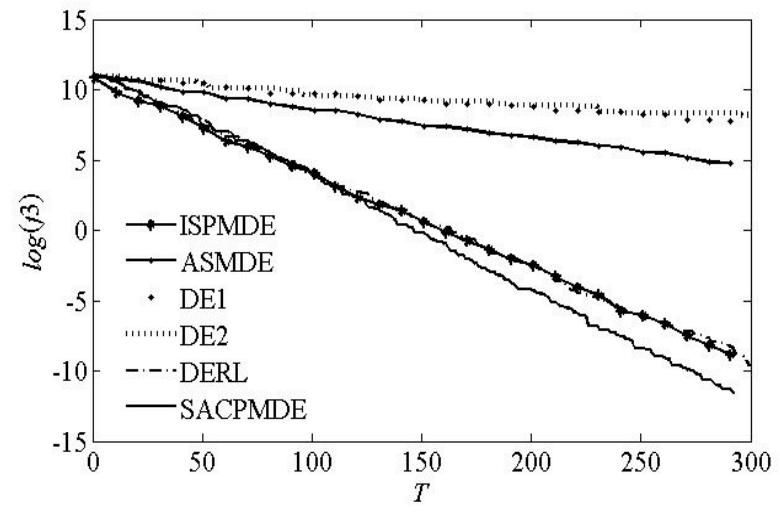

Fig. (6). Convergence curves of the objective function $f 3$.

(1) Test function f1. ISPMDE and ASMDE algorithm performance is closer, DERL convergence value is optimal but it appears to be premature. Compared with other algorithms the optimization effect is not ideal.

(2) Test functions $f 2$. ISPMDE algorithm's performance advantage is obvious. Convergence speed is obviously better than other algorithms. In addition to DERL algorithm, other algorithms are premature. Although DERL is optimal but its speed is slow.

(3) Test functions $f 3$. ISPMDE, ASMDE and SACPMDE algorithm performance is close. SACPMDE is better than the other two algorithms. Although the algorithm is optimized but the speed is slow.

In Table 1, we adopted different algorithms for different types of test functions. We can see that algorithm design is flawed or the target is too much. Algorithm is suitable for the function optimization. Although ISPMDE algorithm for individual test function is slightly lower (basic), the overall performance is stable.

Experiment 2: (ISPMDE-SVM video key frame extraction) video key frame extraction of evaluation index mainly has two indicators: check precision rate and check whole rate. We use standard database TREC2009 video library. The following is to compare different algorithms, check precision rate and check whole rate are testified by the simulation and proper analysis, the results are shown in Figs. (7 and 8).

The results are ideal. It is dependent on the two evaluation indexes. The high rated algorithm for video key frames can be effective identified. Relatively low check precision rate does not mean that the video key frame extraction comprehensive is also low. The low threshold value causes algorithm error and the frames are relatively increased. Similarly, the index of the frame rate does not mean that the algorithm accuracy is higher for the video key frames. Only the check precision rate and the check whole rate of equilibrium can reflect the performance advantage of video extraction algorithm. Figs. (7 and 8) show the algorithm respectively for TREC2009 video library and performance rate:

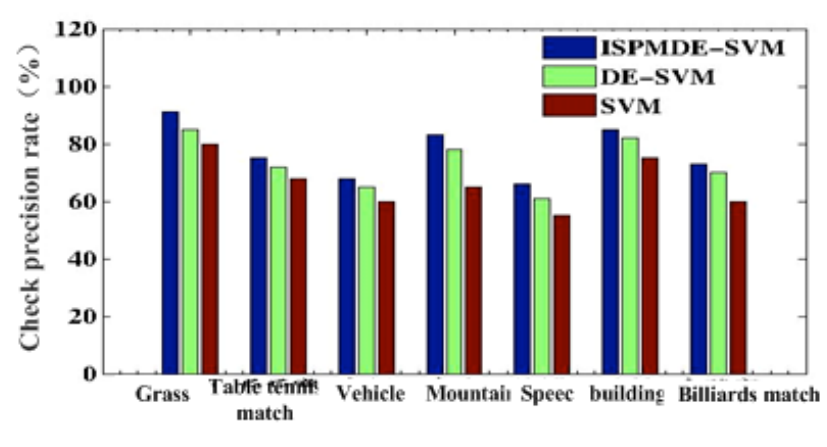

Fig. (7). Check precision rate.

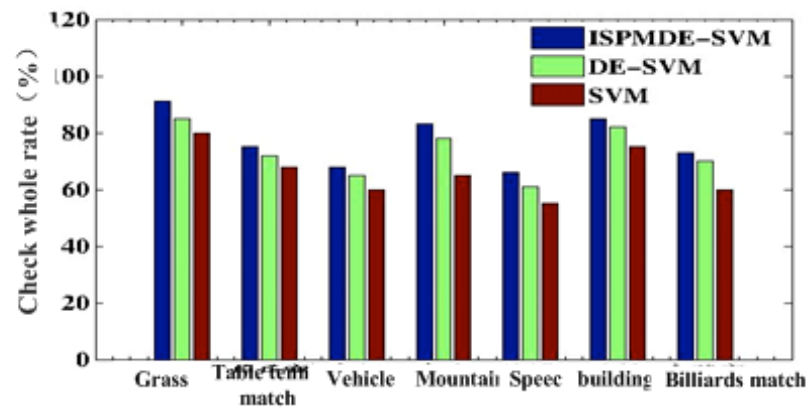

Fig. (8). Check whole rate.

(1) Check precision rate aspects. Among three algorithms for grassland, alpine and building relative single obvious key frame extraction effect is good. The ISPMDE-SVM algorithm overall result is superior to DE- SVM algorithm, and the SVM algorithm of key frame extraction precision result is the worst.

(2) Check entire rate aspects. Three algorithms with grass, table tennis and vehicles are the highest. Overall ISPMDESVM algorithm is superior to DE-SVM algorithm, the video key frame of the SVM algorithm extraction check whole rate of the result is the worst.

Considering film 《Thor II: The Dark World》, we establish a synthetic video clips for algorithm key frame extraction. The accuracy of the simulation test consists of four parts and 256 frames. Its characteristics are dependent on background color. Bigger interference on the front key information leads to higher similarity of the middle lenses (including background). The first shot of the main information is similar degree higher than the middle of the two lenses, and the video shot background difference is bigger. The fourth video shot and the front three video shots are obviously different on the vision, and the background has large difference.

The main design has two aspects: (1) Three video test shots are used for the test of the key frames using the best discrimination ability. (2) The fourth test shots used to test the minimum discrimination performance of three algorithms. To facilitate comparison, we use the video footage of the first frame as the target of retrieval, which use real first frame for the standard of the original video shot, as shown in Fig. (9). It can be seen intuitively. The three shots 

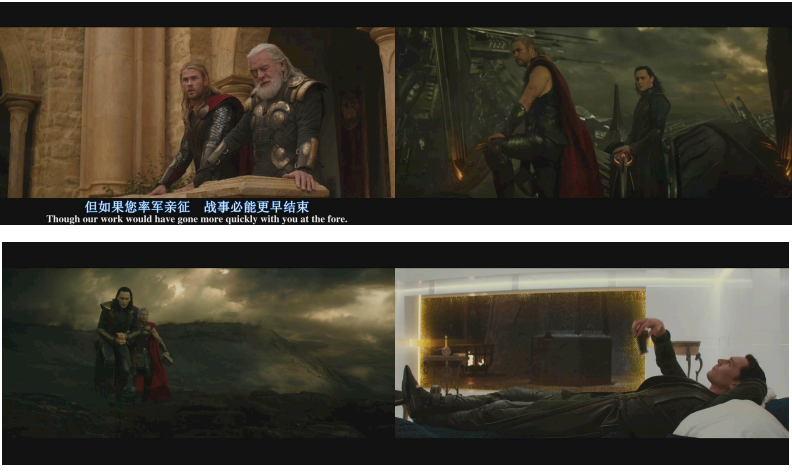

(a) The first frame of the original shots
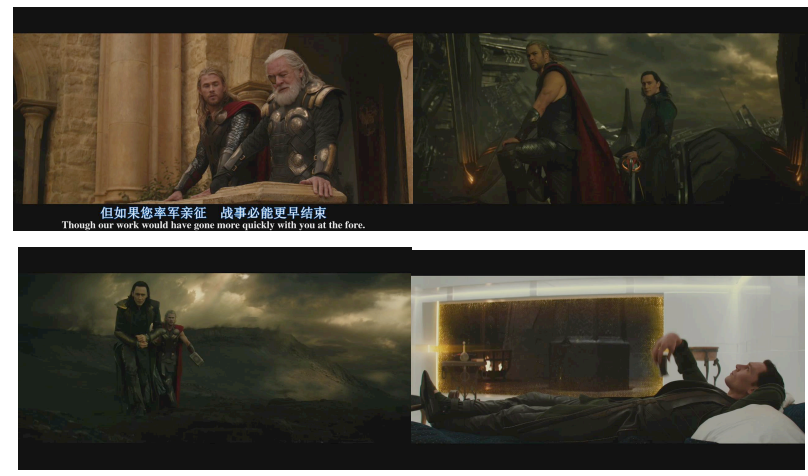

(b) ISPMDE-SVM algorithm to extract the first frame
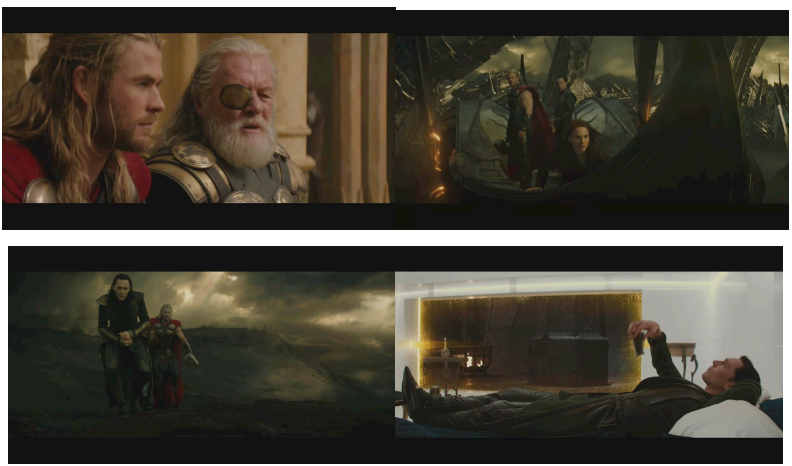

(c) DE-SVM algorithm to extract the first frame
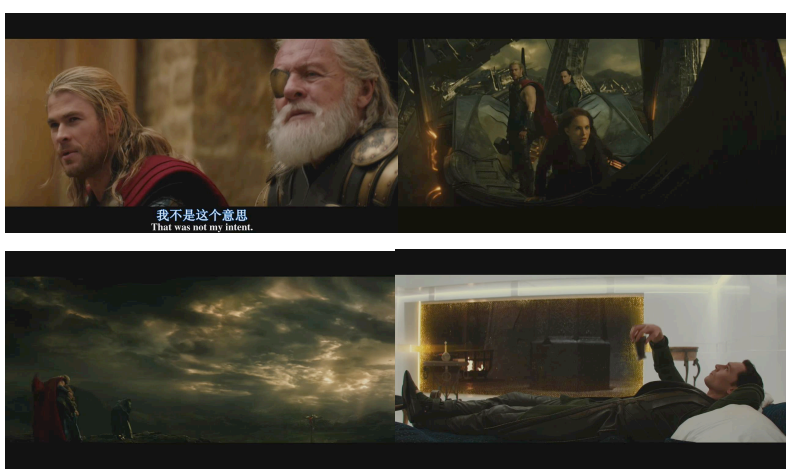

(d) SVM algorithm to extract the first frame

Fig. (9). Movie clips of key frame extraction.

are based on ISPMDE-SVM algorithm. We extract the first frame and the original video of the first frame. The algorithms for the fourth shot are based on the key frames of the first frame. We consider the original shots of the first frame. Conclusion: three algorithms with simple contrast give high accuracy for the extraction of video. When the shots similarity degree are higher (main information and background information), the ISPMDE-SVM video key frame extraction algorithm performance is more stable. The accuracy of extraction is the best. The algorithm is suitable for the practical application.

\section{CONCLUSION}

In this paper we study the video key frame extraction algorithm for big amount of data. The algorithm is simple and efficient for its actual application. Its value is shown from the perspective of minimize the impact on the computing complexity. The angle of mechanism analysis improved the way of mutation. Based on differential evolution algorithm it is the most efficient way and it has barely increased computational time complexity. The algorithm's improvement and efficiency are effective. By means of the test function the simulation show that ISPMDE algorithm has higher convergence speed and accuracy. Finally using SVM for classification algorithm, we extract the video key frame using ISPMDE algorithm. The video test database library and self-built test library of the simulation results show that the algorithm has higher precision rate. The first key frame of video library is selfbuilt with better accurate. The experiments show that ISPMDE-SVM video key frame extraction algorithm is feasible and effective.

\section{CONFLICT OF INTEREST}

The authors confirm that this article content has no conflict of interest.

\section{ACKNOWLEDGEMENTS}

This work was financially supported by Special Fund of XJPCC Youth Science and Technology Innovation (2013CB009, 2014CB004), Key Technology Developing Program of Shihezi University (gxjs2012-zdgg03) and the National Training Programs of Innovation and Entrepreneurship for Undergraduates (201410759030).

\section{REFERENCES}

[1] P. Kaelo, and M.M. Ali, "A numerical study of some modified differential evolution algorithms," European Journal of Operational Research, vol. 169, pp. 1176-1184, 2006.

[2] S. Feng, and R.A. Manmatha, "Discrete direct retrieval model for image and video retrieval," In: Proceedings of the 2008 International Conference on Content-based Image and Video Retrieval (CIVR'08), Niagara Falls, Ontario, ACM: Canada, 2008, pp. 427-435.

[3] C. Grana, R. Vezzani, and R. Cucchiara, "Enhancing HSV histograms with achromatic points detection for video retrieval," In: Proceedings of the $6^{\text {th }}$ International Conference on Image and Video Retrieval (CIVR'07), Amsterdam, ACM: The Netherlands, 2007, pp. 302-308.

[4] G.T. Papadopoulos, V. Mezaris, and I. Kompatsiaris, "Accumulated motion energy fields estimation and representation for semantic event detection," In: Proceedings of the 2008 International Conference on Content-based Image and Video Retrieval (CIVR'08), Niagara Falls, Ontario, ACM: Canada, 2008, pp. 221-230. 
[5] A.H. Wright, Genetic Algorithms for Real Parameter Optimization. Foundations of Genetic Algorithms, Morgan Kaufmann: Rawlins GJEEDSCA, 1991, pp. 205-218.

[6] X.G. Shao, H.Z. Yang, and G. Chen, "Parameters selection and application of support vector machines based on particle swarm optimization algorithm," Control Theory \& Applications, vol. 23, no. 5, pp. 740-748, 2006.

[7] S.P. Yong, D. D. Jeremiah, and K. P. Martin, "Wildlife video keyframe extraction based on novelty detection in semantic context," Multimedia Tools and Applications, vol. 62, no. 2, pp. 359-376, 2013.

[8] L.H. Wu, Y. N. Wang, and S.W. Zhou, "Research and application of pseudo parallel differential evolution algorithm with dual subpopulations," Control Theory \& Applications, vol. 24, no. 3, pp. 453-458, 2007.

[9] W. Barhoumi, and E. Zagrouba, "On-the-fly extraction of key frames for efficient video summarization," AASRI Procedia, vol. 4 , pp. 78-84, 2013.

[10] X.M. Liu, A.M. Hao, and D. Zhao, "Optimization-based key frame extraction for motion capture animation," The Visual Computer, vol. 29, no. 1, pp. 85-95, 2013.

[11] K. Sanjay, K. R. Panda, and S. Ananda, "Chowdhury Video key frame extraction through dynamic Delaunay clustering with a structural constraint," Journal of Visual Communication and Image Representation, vol. 24, no. 7, pp. 1212-1227, 2013.

[12] G.L. Guan, "Key-point based key-frame selection," IEEE Circuits and Systems Society, vol. 23, no. 4, pp. 729-734, 2013.

(C) Ronghua and Changzheng; Licensee Bentham Open.

This is an open access article licensed under the terms of the Creative Commons Attribution Non-Commercial License (http://creativecommons.org/licenses/by-nc/4.0/) which permits unrestricted, non-commercial use, distribution and reproduction in any medium, provided the work is properly cited. 